\title{
Driver behavior in mixed and virtual reality - a comparative study
}

\author{
B. Blissing, F. Bruzelius, and O. Eriksson
}

Swedish National Road and Transport Research Institute; SE-58195 Linköping; Sweden, e-mail: \{bjorn.blissing, fredrik.bruzelius, olle.eriksson\}@ vti.se

\begin{abstract}
This paper presents a comparative study of driving behavior when using different virtual reality modes. Test subjects were exposed to mixed, virtual, and real reality using a head mounted display capable of video see-through, while performing a simple driving task. The driving behavior was quantified in steering and acceleration/deceleration activities, divided into local and global components. There was a distinct effect of wearing a head mounted display, which affected all measured variables. Results show that average speed was the most significant difference between mixed and virtual reality, while the steering behavior was consistent between modes. All subjects but one were able to successfully complete the driving task, suggesting that virtual driving could be a potential complement to driving simulators.
\end{abstract}

Keywords: Mixed Reality, Virtual Reality, Head Mounted Display, Driver Behavior

\section{Introduction}

Driving simulators offer an almost completely controlled environment with high repeatability, reproducibility and flexibility in terms of capability to realize complex and dangerous scenarios. Studies can be performed that are hard or impossible to perform in real vehicles even on test tracks. However, validity of the test subjects behavior and reactions in the simulator may be questioned due to incomplete, incorrect or missing feedback cues [Kem03]. One such mismatch in cues is an effect of the limitations of the motion system in driving simulators.

Using a vehicle fitted with an augmented, mixed or virtual reality visual system can be a potential alternative to using driving simulators in driver-vehicle interaction studies. One of the benefits would be validity of the motion feedback that the drivers are experiencing, as they are exposed to the real accelerations. Other benefits are lower investment costs, flexibility in terms of installation and ease of operation.

The performance and behavior of mixed and virtual reality systems are, to a large extent, determined by the display techniques used to present the computer generated graphics. A wide range of display techniques can be used to create the image for augmented, mixed or virtual reality. One option is to use a head mounted display (HMD) to present the visual cues to the driver, using either an optical see-through HMD [Boc07], a video see-through HMD [Ber13] or using an opaque HMD for pure virtual worlds [Kar13]. Another option is to use the windshield as a projection area, either using the windshield as an optical combiner to achieve optical see-through [Par14], having video cameras facing forward and display the augmented image on screens mounted in front of the windshield [Uch15], or using the windshield as an opaque projection screen [Rie15] (See table 1). The technical advantages and disadvantages of the different display techniques are further detailed in [Bli13].

This study focuses on a HMD solution developed in [Bli15] to represent mixed and virtual reality. Driver behavior using this solution in respect to latency have previously been evaluated [Bli16]. This mixed reality solution superimposes virtual objects in the real environment, as opposed to the solution used in [Ber13], where only the interior of the vehicle is real and a completely virtual environment is presented as a replacement for the view from the windshield.

Using these techniques as a complement to driving simulators require an understanding of how drivers are affected by the selected mode of virtuality. We present a comparative study of driver behavior using two HMD based setups; video see-through (VST) and pure virtual reality (VR). The underlying questions to be addressed in this study are,

1. How does driving behavior change between normal driving with a direct view of the environment compared to driving while wearing a HMD?

2. How does driving behavior change between the different VR-modes? Is one of the modes preferable over the others with respect to driving behavior?

\section{Methodology}

The test subjects were instructed to perform simple driving tasks at low speed, while vehicle data were recorded. The study was performed as a within-group study to minimize any interacting effects between the studied VR-modes. 
Table 1: Previous research with different modes of Virtual- and Mixed Reality.

\begin{tabular}{|c|c|c|c|}
\cline { 3 - 4 } \multicolumn{2}{c|}{} & Fixed Display & Head Mounted Display \\
\hline Virtual Reality & Opaque & {$[$ Rie15] } & {$[$ Kar13] } \\
\hline \multirow{2}{*}{ Mixed Reality } & Optical See-through & {$[$ Par14] } & {$[$ Boc07] } \\
\cline { 2 - 4 } & Video See-through & {$[$ Uch15] } & {$[$ Ber13] } \\
\hline
\end{tabular}

\section{Subjects}

A group of 22 participants were recruited among the staff at VTI (14 men and 8 women). All participants were naïve to the details of the experiment and none of them were given any compensation for their participation. The implication of selecting VTI staff members only for the experiment is believed to be minor as the they came from varied departments within $\mathrm{VTI}$, and have different professions, age, and driving experience. None of them were trained test drivers. Their age ranged from 22 to 64 (mean age 37) and their annual mileage ranged from 5000 to $15000 \mathrm{~km}$ (mean $10000 \mathrm{~km}$ ).

The participants were required to have a valid drivers license and being able to drive without glasses. The last requirement was due to space constraints inside the HMD, leaving no space for glasses between the HMD and the eyes. Before the study the participants were asked to sign a form of informed consent, explicitly stating the right to abort at any time during the experiment.

\section{Apparatus}

A Volvo V70 with automatic gearbox was equipped with a custom mixed reality solution [Bli15] and used as test platform. The solution consisted of an Oculus Rift Development Kit 2 HMD with two IDS uEye UI-3240CP-C cameras attached (see Figure 1). The cameras are able to capture full color images with a global shutter in $1280 \times 1024$ pixels at $60 \mathrm{~Hz}$, i.e. $16.6 \mathrm{~ms}$ per frame. The images were rectified via an OpenGL shader to correct for any optical distortion and then sent to the 3D-rendering engine.

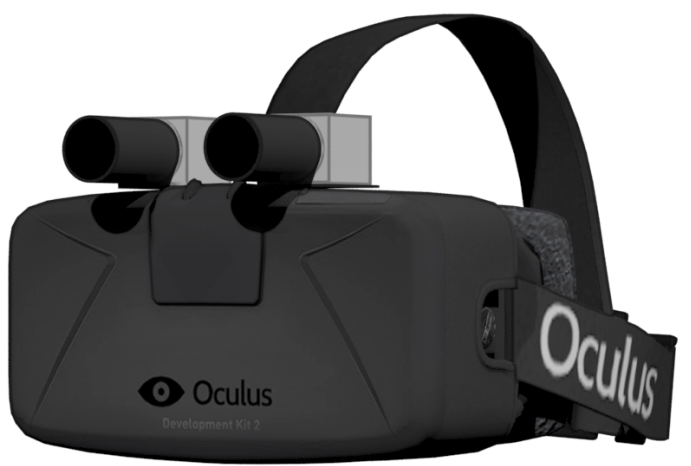

Figure 1: Oculus Rift Development Kit 2 with high resolution cameras mounted on top.

The HMD is capable of rendering at $75 \mathrm{~Hz}$, i.e. $13.3 \mathrm{~ms}$ per frame. Since the 3D-rendering engine is running asynchronously with the camera capture the camera images had to be buffered to avoid image tearing. Regrettable, this buffering can increase the visual latency with up to one frame, depending on the timing of when the camera images are required by the rendering engine. The measured latency in the camera is $51 \pm 25 \mathrm{~ms}$. The rendering engine is also using double buffering, which can delay the image in the HMD with yet another frame. Together with the screen scanout time and graphics driver overhead the resulting total latency in the opaque virtual reality system is $44 \pm 20 \mathrm{~ms}$. Combining these results, the total latency for the mixed reality system can be estimated to be in the order of $100 \mathrm{~ms}$.

The cameras were mounted flush with the profile of the device to make the assembly strong. This is why the surface of the camera images in Figure 2 are rotated a few degrees along the optical axis. The optics used for the cameras limited the monocular field of view to $62^{\circ}$ horizontal and $48^{\circ}$ vertical. This view is narrower compared to what is achievable in the HMD, which is specified at $90^{\circ}$ horizontal and $100^{\circ}$ vertical. Hence, whenever the cameras were used, a more narrow field of view was obtained. The difference in field of view is visualized in Figure 3.

The test vehicle was fitted with a GPS-system with an inertial measuring unit capable of recording linear accelerations and rotational velocities around all three axes. The GPS system, a Racelogic VBOX with $100 \mathrm{~Hz}$ sample rate, was used in a RTK configuration and with a base station resulting in a resolution of $1 \mathrm{~cm}$ and $0.01 \mathrm{~km} / \mathrm{h}$, according to the instrument supplier.

\section{Registration Errors}

One of the largest problems with augmented and mixed reality is the failure to correctly superimpose the computer generated objects onto the user's view of the real world. These types of errors typically occur due to system delays, tracker error or errors in calibration of the HMD. The system delays are usually the largest source of errors [Hol97]. Especially HMDs with optical see-through are very sensitive to system delays as they present the view of the real world without any delay, while the computer generated objects have some render delay. When using a HMD with video see-through, the view of the real world has an additional delay due to image processing pipeline, which may compensate for some of the render delay. There is also the possibility to correct for the registration errors using feature detection [Baj95].

The visual latency in the current HMD setup resulted in noticeable registration errors. There were also noticeable misregistrations due to lack of tracker accuracy. To be able to mitigate the effects of these types of errors some form of image based correction would be necessary. This would also require either fitting the environment with good tracking targets or the employment of computational heavy algorithms. Fitting the environment with additional targets could potentially distract the drivers and using computational heavy algorithms would increase the latency even more. 

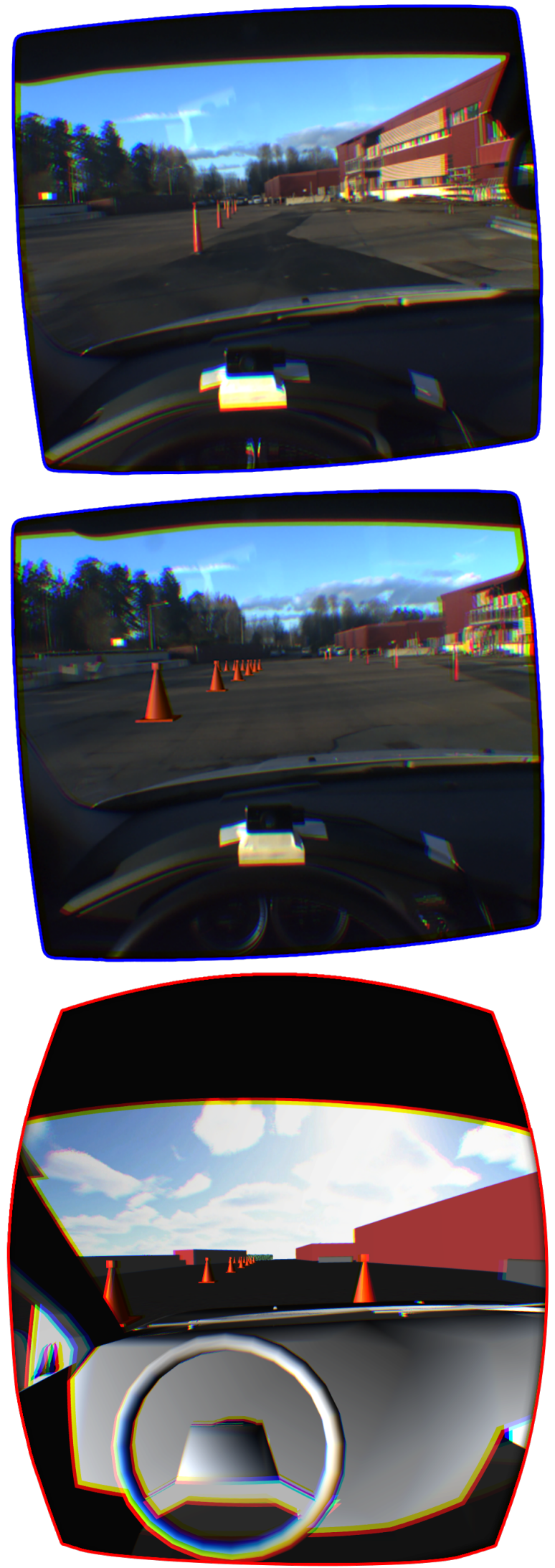

Figure 2: Screen shots of the video see-through view with real cones (top), the video see-through view with virtual cones (middle) and virtual world (bottom). Note that the video see-through images are scaled up for clarity, since their field of view are narrower as seen in Figure 3.

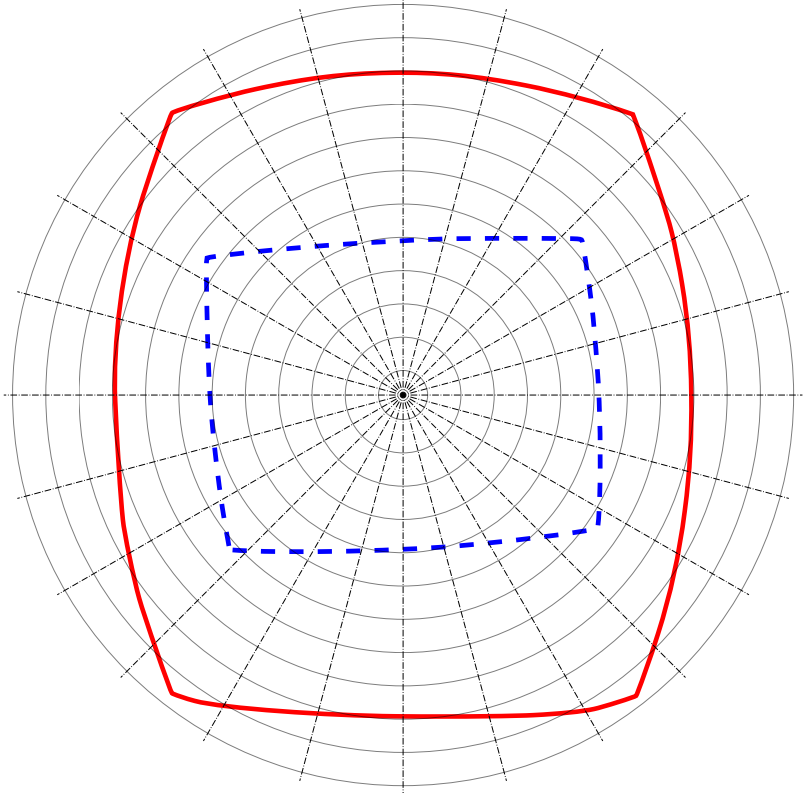

Figure 3: Difference in monocular field of view between virtual mode (red) and video see-through mode (blue dashed). Each concentric circle represents $10^{\circ}$.

\section{Procedure}

The participants were asked to drive a slalom course in their own pace under 4 different modes:

Video See-Through-Real Reality (VST-RR) -

Using video see-through head mounted display which only feed the video stream without any overlays. The slalom course uses real cones.

Video See-Through-Mixed Reality (VST-MR) Using video see-through head mounted display in which virtual cones are superimposed in the video stream.

Virtual Reality (VR) - Using an opaque head mounted display which presents a completely virtual world. This world has been constructed to be similar to the real world.

Direct View (DV) - Using direct view of the environment, i.e. driving without any head mounted display.

The slalom course consisted of five cones placed ten meters apart. Another line of cones were positioned five meters after the last cone of the slalom track to stop the participants from exiting the test area (see figure 4).

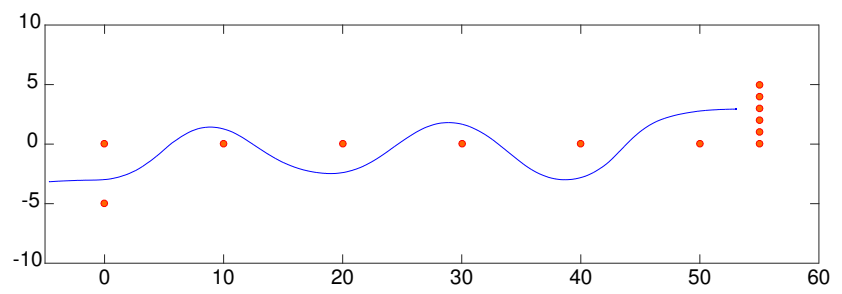

Figure 4: The test track setup and suggested path.

As driving behavior varies between individuals, the study was performed using a within-subject design. Each person started with driving the slalom course three times with direct view to familiarize them to 
the vehicle, as well as making sure that they understood the driving task clearly. After these training runs, the participants were subjected to the different VR-modes. Each condition was repeated three times. The conditions were run in a balanced order for the different subjects to minimize potential interaction effects. Finally, all subjects drove without the HMD three more times. These final runs are used as a comparative baseline for all measurements.

After each run, the participants were asked to self assess both the difficulty of the driving task and to rate their own performance. The self assessment was made on a scale with seven steps, going from Very Easy to Very Hard for difficulty and from Very Bad to Very Good for performance.

\section{Objective measurements}

The GPS and IMU signals recorded during the test runs were used to objectively quantify the driver behavior. The measures, see table 2 , were chosen to reflect two dimensions of the driver behavior; the lo$\mathrm{cal} / \mathrm{global}$ and the lateral/longitudinal behavior. The lateral/longitudinal dimension corresponds to steering and accelerator/brake pedal activities, while the local/global differentiates between specific corrections versus general drive style throughout the test run. The four measures are further explained below.

Table 2: Group of measurements

\begin{tabular}{|l|cc|}
\cline { 2 - 3 } \multicolumn{1}{c|}{} & \multicolumn{1}{c|}{ Local } & Global \\
\hline $\begin{array}{l}\text { Longitudinal } \\
\text { Lateral }\end{array}$ & $\begin{array}{c}\text { Acceleration changes } \\
\text { Maximum curvature }\end{array}$ & $\begin{array}{c}\text { Time to completion } \\
\text { Lateral deviation }\end{array}$ \\
\hline
\end{tabular}

Time to completion $-T_{c}$, is the time used from passing the first cone until passing the last cone. Since the participants were not instructed to maintain a fixed speed, this will be a measure of how comfortable they were in current VR-mode. The hypothesis is that this measure will increase as the participants decrease their speed to compensate for any discomfort with the visual impression.

Acceleration changes $-A_{c}$, is defined as the number of acceleration changes made during the drive, i.e. the jerkiness. This measures how often the participants needed to make velocity corrections. The hypothesis is that jerkiness will increase when the participants adjust the velocity to compensate for discomfort or distrust of the visual impression along the test run.

Maximum curvature $-M_{c}$, is defined as the maximum value of the ratio between the vehicle yaw rate $\psi$ and the vehicle velocity $v_{x}$,

$$
M_{c}=\max _{t}\left(\frac{\psi}{v_{x}}\right)
$$

The fraction above corresponds to the curvature of a steady state motion vehicle. A higher value of this measure indicates that the driver is steering more and driving in a smaller radius. The maximum value of this curvature will be a measure of how much the driver needs to steer during the worst situation along the test run. The hypothesis is that this measure will increase if any of the VR-modes are perceived as more difficult.
Lateral deviation $-L_{m}$, is calculated as the Root Mean Square (RMS) of the lateral position (perpendicular to the cone slalom course) of the vehicle trajectory $r$ :

$$
\begin{gathered}
m_{r}=\frac{1}{L} \int_{L} r(s) d s \\
L_{m}=\sqrt{\int_{L}\left(r(s)-m_{r}\right)^{2} d s}
\end{gathered}
$$

where $s$ is the longitudinal position of the trajectory (in line with the slalom course), and $L$ is the total longitudinal length of the track. The hypothesis is that this measure will capture the overall lateral behavior of driving and the lateral margins to the cones in the track on average. The hypothesis is that the subjects would compensate with greater margins to the cones if any of the VR-modes were deemed more difficult.

\section{Results}

During the tests, there were problems with the communication between the base station and the GPS system in the test vehicle. Consequently the precision of the measurements drops radically and the position signal can skip large distances between two samples. This makes the data useless in this context. Hence, objective data from three (3) of the participants were unusable and had to be removed from the analysis, although the data from the self assessment were still possible to use.

\section{Motion sickness}

Since the test consisted of many short driving tasks the use of the standard Simulator Sickness Questionnaire (SSQ) was deemed unusable. One participant had to abort the test due to motion sickness, and data from this person have been excluded from the analysis. This person developed motion sickness quickly and elected to abort after the forth run, i.e. only one run with the HMD.

\section{Data analysis}

The statistical model used for this experiment is $Y_{i j k}=\alpha_{i}+\beta_{j}+C_{k}+\alpha C_{i k}+\epsilon_{i j k}$ where $\alpha$ is the fixed factor VR-mode, $\beta$ is the fixed factor run and $C$ is the random factor subject. The model was analyzed with a three way ANOVA. Pairwise comparisons between levels on the fixed factors were performed and corrected for multiple comparisons by the Tukey method. The variance components were estimated for the random factors. The results of the ANOVA were summarized with $\mathrm{P}$-values in Table 3.

\section{Fixed factor levels}

Table 4 shows the means for the fixed factor levels. The means are expressed as least squares means and do not only use the data, but also the model to adjust for unbalanced missing data. Comparisons between pairs of fixed factor levels are also included by showing, with letters, which group(s) a mean belongs to. Means that do not share a letter are significantly different. 
Table 3: P-values when testing that there are no factor effects and no interaction between subject and VR-mode

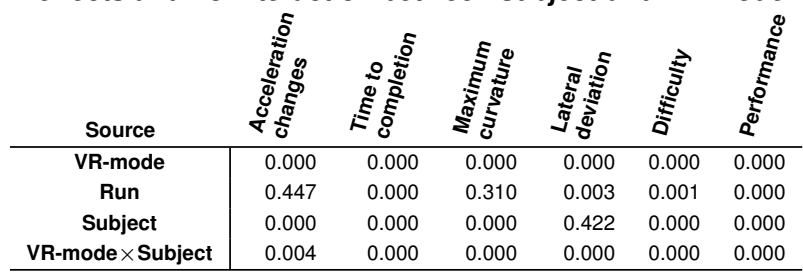

Table 4: Means and pairwise comparisons for fixed factor levels.

\begin{tabular}{|c|c|c|c|c|c|c|c|c|c|c|c|c|}
\hline & 造 & & हू & & 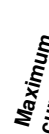 & & ฐ্ণ & & : & & ڤ & \\
\hline VR-mode & & & & & & & & & & & & \\
\hline VST-RR & 8.94 & A & 21.0 & A & 7.77 & A & 10.9 & A & 3.92 & A & 4.27 & A \\
\hline VST-MR & 9.64 & A & 25.4 & B & 9.96 & B & 12.1 & A & 5.16 & B & 3.68 & A \\
\hline$V R$ & 8.82 & A & 22.0 & A & 9.52 & B & 12.2 & A & 4.13 & $A$ & 4.19 & A \\
\hline$D V$ & 6.52 & B & 14.7 & C & 6.78 & C & 8.3 & B & 1.51 & C & 6.25 & B \\
\hline Run & & & & & & & & & & & & \\
\hline 1 & 8.70 & A & 21.7 & A & 8.47 & A & 11.1 & A & 3.87 & A & 4.32 & A \\
\hline 2 & 8.39 & A & 20.7 & B & 8.59 & A & 11.0 & A & 3.68 & $A B$ & 4.66 & B \\
\hline 3 & 8.35 & A & 19.9 & C & 8.47 & A & 10.5 & B & 3.49 & B & 4.82 & B \\
\hline
\end{tabular}

\section{Estimation of variance components}

Table 5 shows information about the size of the variation between random factor levels, and also the size of the residual variation. Interaction between VRmode and subject is random because subject has random factor levels.

Table 5: Variance components

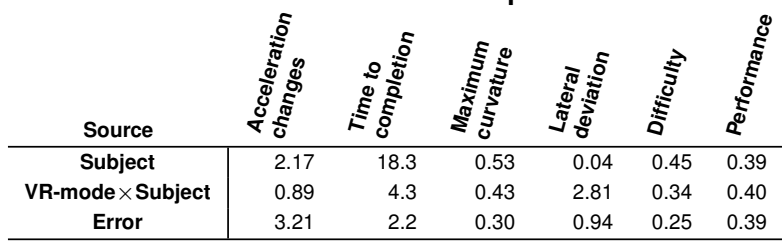

\section{Summary of the data analysis}

It may be obvious that there is a variation between subjects, and that a formal test to show such variation is not very important. However, the effect of less interesting factors and their contribution to the variation must be modeled and properly handled. Otherwise the error term for the other test will not be correct.

When looking at comparisons between levels of $V R$ mode (Table 4), direct view differs significantly from VR-modes in each response variable. The difference between the individual modes VST-RR, VST-MR and VR do not show the same pattern for each response variable, but in most cases modes VST-RR and VR can not be separated while VST-MR can be separated from VST-RR and VR for some of the response variables.

It appears that some learning effects between runs are present in Time to completion, for the rest of the response variables the differences between runs are small and may not be very important to study in detail. As can be seen in Table 5, the response variables behave quite differently in respect to the largest variation source. The size of the variation between levels of a fixed factor can also be expressed as a variance, making it possible to compare VR-mode as a variation source to the factors in Table 5:

Acceleration changes The largest source of variation is the unexplained residual variation, followed by the variation between subjects.

Time to completion The largest variation source is Subject followed by difference between levels of $V R$-mode. This is expected since the subjects selected velocity according to their own comfort level, but all were forced to adapt their velocity to the current VR-mode.

Lateral deviation The largest variation sources are $V R$-mode and VR-mode $\times$ Subject. This is the only response variable where the interaction is comparably large. The variation between VR-mode is comparable to the variation between subjects but the pattern in the variation between subjects changes between levels of VR-mode.

For all other variables the selected VR-mode is the largest source of variation.

\section{Deviations from the used model}

For Difficulty, interaction between Subject and Run was significant $(P=0.039)$ with estimated variance component 0.03 . For Time to completion, interaction between VR-mode and Run was significant $(P=$ 0.005 ). As can be seen in Table 6 , only negligible improvements can be seen between runs for DV and VST-RR, while some improvement can be seen for VR between the first and second run. For VST-MR improvements can be seen between all three runs.

Table 6: Means for combinations of VR-mode and Run for Time to completion

\begin{tabular}{|c|c|c|c|}
\hline & $\hat{s}$ & $\stackrel{\sim}{s}$ & $\stackrel{m}{\Sigma}_{\mathbb{E}}^{\infty}$ \\
\hline \multicolumn{4}{|l|}{ VR-Mode } \\
\hline VST-RR & 21.77 & 21.10 & 20.26 \\
\hline VST-MR & 26.93 & 25.44 & 23.70 \\
\hline VR & 23.39 & 21.56 & 21.11 \\
\hline DV & 14.85 & 14.62 & 14.50 \\
\hline
\end{tabular}

\section{Discussion}

Introducing a HMD based visual system to a driver may affect the driving behavior compared to driving with direct view of the environment.

In a previous study the effect of latency on driving behavior was studied using a similar setup delaying the visual information to the driver [Bli16]. It was concluded that the drivers were able to compensate for latency to a large extent, even for large latencies, but altered their behavior with greater margins and more correcting actions.

In [Boc07], an optical see-through HMD was used and a couple of common driving maneuvers were validated. Most behaviors were considered similar, except behaviors dependent on reaction time.

In [Kar13] a opaque HMD was used. Only the maximum steering behavior, maximum brake pressure and maximum deceleration showed absolute validity in this study. They also mention increased reaction time leading to changed absolute longitudinal behavior, although the relative behavior had the same magnitude. 
In this study, we found that the participants altered their brake and accelerator behavior when using the HMD, compared to the direct view case. On average they drove $35 \%$ slower while wearing the HMD. The differences between the different VR-modes were smaller for both acceleration changes and for the average speed. Only mixed reality mode differs with a significantly lower average speed compared to the other modes.

For the steering behavior a similar difference could be observed as for the longitudinal case. The direct view runs without the HMD differs compared to those with the HMD for both minimum radii and average lateral margin to the cones. For the other VR-modes, sharper turns were made with mixed and virtual reality modes, while the average lateral margin was not significantly different to all cases with the HMD.

The self-assessment measures are in-line with the other measures regarding the difference between wearing and not wearing the HMD, but mixed reality is perceived as the most difficult mode of virtuality. This is probably due to the narrower field of view as well as the noticeable registration errors in the current VST HMD.

Most of the measures changed for each test run, indicating a learning effect. This effect was significant for the average speed in general and most noticeable for the mixed and virtual reality modes. The learning effect was also significant for the self-assessments and the average steering behavior, but with smaller differences than for the average speed.

Notably, most of the measures between virtual and mixed reality were not significantly different, with the exception of average speed. The similarity between the two modes indicate that the narrower field of view did not affect steering behavior.

It can also be seen that the difference between the different VR-modes had a larger effect on the driving behavior compared to introducing substantial latency in the visual system, see [Bli16]. This, together with the difference in driving behavior for mixed reality, can be seen as an indication that there could be an advantage in sacrificing latency to reduce registration errors in mixed reality.

The learning effects noted in this study raise the question whether this could be used to train subjects to use VR-mode solutions for improved validity. Studies that involve extended use of the VR-mode solutions would be required for studying potential learning effects in more detail.

\section{Conclusions}

All subjects but one were able to drive in all conditions even though there was a clear effect of using the HMD based visual system compared to a direct view. This work illustrates the importance of selecting the proper type of technology for the desired scenarios by quantifying the difference in driving behavior for the different VR-modes. Currently, the VR solution is deemed better than the VST solution.

The main challenges for future development are to reduce latency and improve tracking. The current GPS-system and IMU based tracking system do not provide enough accuracy to be used as input to a mixed reality solution. To eliminate the registration errors, some form of image based tracking technology is probably the only possible solution.

\section{Acknowledgments}

This project is mainly funded by the VINNOVA/FFI project Next Generation Test Methods for Active Safety Functions. Additional funding have also been provided by the Swedish National Road and Transport Research Institute via the strategic research area TRENoP.

\section{References}

M. Bajura and U. Neumann, Dynamic registration correction in video-based augmented reality systems, IEEE Computer Graphics and Applications, vol. 15(5): 52-60, 1995.

G. Berg, T. Millhoff and B. Färber, Vehicle in the Loop - Zurück zur erweiterten Realität mittels video-see-through, in Fahrer im 21. Jahrhundert, vol. 2205, 225-236, VDI-Verlag, Düsseldorf, 2013.

B. Blissing, F. Bruzelius and J. Ölvander, Augmented and Mixed Reality as a tool for evaluation of Vehicle Active Safety Systems, in Proceedings of the 4th International Conference on Road Safety and Simulation, Roma Tre University, Rome, Italy, 2013, ISBN 978-1-4951-7445-2.

B. Blissing and F. Bruzelius, A Technical Platform Using Augmented Reality For Active Safety Testing, in Proceedings of the 5th International Conference on Road Safety and Simulation, 793803, University of Central Florida, Orlando, FL, USA, 2015, ISBN 978-1-4951-7445-2.

B. Blissing, F. Bruzelius and O. Eriksson, Effects of visual latency on vehicle driving behavior, ACM Transactions on Applied Perception, 2016, conditionally accepted.

T. Bock, M. Maurer and G. Färber, Validation of the Vehicle in the Loop (VIL) - A milestone for the simulation of driver assistance systems, in Proceedings of the 2007 IEEE Intelligent Vehicles Symposium, 219-224, IEEE, Istanbul, Turkey, 2007, ISBN 1-4244-1067-3.

R. L. Holloway, Registration error analysis for augmented reality, Presence: Teleoperators and Virtual Environments, vol. 6(4): 413-432, 1997.

I. Karl, G. Berg, F. Ruger and B. Färber, Driving Behavior and Simulator Sickness While Driving the Vehicle in the Loop: Validation of Longitudinal Driving Behavior, IEEE Intelligent Transportation Systems Magazine, vol. 5(1): 42-57, 2013.

A. Kemeny and F. Panerai, Evaluating perception in driving simulation experiments, Trends in Cognitive Sciences, vol. 7(1): 3137, 2003.

H. Park and K. Kim, AR-Based Vehicular Safety Information System for Forward Collision Warning, in Virtual, Augmented and Mixed Reality. Applications of Virtual and Augmented Reality, 435-442, Springer International Publishing, 2014, ISBN 978-3319-07463-4.

B. Riedl and B. Färber, Evaluation of a new projection concept for the Vehicle in the Loop (VIL) driving simulator, in Proceedings of Driving Simulation Conference 2015 Europe, 225226, Tübingen, Germany, 2015, ISBN 978-3-9813099-3-5.

N. Uchida, T. Tagawa and K. Sato, Development of an instrumented vehicle with Augmented Reality (AR) for driver performance evaluation, in Proceedings of the 3rd International Symposium on Future Active Safety Technology Towards zero traffic accidents, 2015, 489-492, Chalmers University of Technology, Gothenburg, Sweden, 2015. 